\title{
0 uso da equoterapia como recurso terapêutico para melhora do equilíbrio estático em indivíduos da terceira idade
}

The use of hippotherapy as therapeutic resource to improve the static balance in elderly individuals

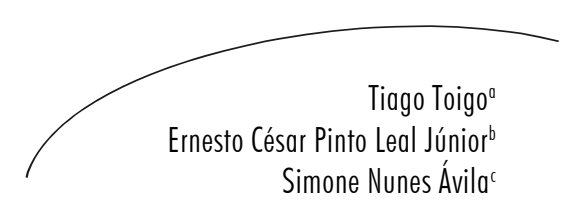

\section{Resumo}

Objetivo: Mensurar a melhora do equilíbrio estático em indivíduos da terceira idade com a prática da equoterapia. Materiais e métodos: estudo experimental, clínico, pré e pós-teste. Dez indivíduos do sexo feminino, entre 60 e 74 anos, residentes na cidade de Caxias do Sul - RS, foram avaliados na estabilometria, pré e pós-aplicação do programa de atendimento equoterapêutico. O mesmo constituía-se de oito sessões de equoterapia, duas vezes por semana, com a duração de 30 minutos cada sessão. Os dados foram analisados por meio dos valores obtidos de velocidade média de deslocamento do CP (Centro de Pressão), deslocamento médio no eixo $\mathrm{X}$ do CP e deslocamento médio no eixo Y do CP. Também foi utilizado o teste estatístico T-Student. O nível de significância aceito foi de $\mathrm{p}<0,05$.

Resultados: Na comparação dos resultados pré e pós-equoterapia, observou-se diferença estatística significativa $(\mathrm{p}=0,002)$ na direção ântero-posterior. Na direção médio-lateral e velocidade de deslocamento, observou-se diferença não-significativa: $(p=0,603)$ e $(p=0,414)$, respectivamente. Conclusão: Indivíduos da terceira idade saudáveis possuem alterações no equilíbrio. A equoterapia foi capaz de melhorar o equilíbrio estático e, consequentemente, diminuir a possibilidade de queda.

\author{
Palavras-chave: \\ Fisioterapia \\ (Especialidade). \\ Modalidades de \\ Fisioterapia. \\ Equilíbrio \\ Musculosquelético. \\ Técnicas de \\ exercício e de \\ movimento. \\ Epidemiologia \\ Experimental. \\ Mulheres. Meia- \\ idade. Idoso. Caxias \\ do Sul, RS
}

\footnotetext{
Universidade de Caxias do Sul

Curso de Fisioterapia

Caxias do Sul, RS, Brasil

Correspondência / Correspondence

Simone Nunes Ávila

Av. Borges de Medeiros, 907/91 - Centro

90020-025 - Porto Alegre, RS, Brasil

E-mails: snavila@ucs.br; simoneavila10@brturbo.com.br
} 


\section{Abstract}

Objective: To measure the improvement of static balance in elderly individuals, through hippotherapy practive. Materials and Methods: Experimental study, clinical, pre and post-test. Ten female individuals, aged between 60 and 74 years, living in Caxias do Sul, State of Rio Grande do Sul,, were evaluated in the stabilometric, before and after the application of the physiotherapeutic attendance program. This consisted of eight sessions of hippotherapy, twice a week, with 30 minutes each. Data were analyzed by means of average speed of displacement of CP (center of pressure), mean displacement of $\mathrm{X}$ axle of the $\mathrm{CP}$ and mean displacement of $\mathrm{Y}$ axle of the CP. The statistic test of T-student was also used. The significance level accepted was $\mathrm{P}<0,05$. Results: In the comparison of results before and after hippotherapy, it was observed significative difference $(\mathrm{P}=0,002)$ in the anteroposterior direction. In the medium-lateral direction and displacement speed it was

Key words: Physical Therapy (Specialty). Physical Therapy Modalities.

Musculoskeletal Equilibrium. Exercise movement techniques. Epidemiology Experimental. Women . Middle aged. Aged. Caxias do Sul City, RS observed a non-significative difference $(\mathrm{P}=0,603)$ and $(\mathrm{P}=0,414)$, respectively. Conclusion: Healthy elderly people have alterations in balance. Hippotherapy was able to improve the static balance and, consequently, to reduce the risk of fall.

\section{INTRODUÇÃO}

O envelhecimento é a diminuição da reserva funcional, com a diminuição da resistência às agressões e aumento do risco de morte. É um processo contínuo, geneticamente programado, dentro do ciclo natural da vida. Segundo levantamento de dados do IBGE, ${ }^{1}$ a população brasileira é constituída por 10,2\% de indivíduos acima de 60 anos de idade. Nas próximas décadas, o número de idosos na população tende a crescer no mundo todo. Em 2025, a população atingirá $14 \%$ de indivíduos idosos, colocando o Brasil em sexto lugar mundial em número de habitantes com mais de 60 anos de idade. ${ }^{2}$

Ao saber desse prenúncio do crescimento populacional, e as modificações funcionais e estruturais do organismo desses indivíduos, demonstrou-se certa preocupação quanto ao desequilíbrio e às conseqüências que possam interferir no envelhecimento com qualidade. Analisando essas informações e considerando o desequilíbrio um dos principais fatores que limitam hoje a vida do idoso, podendo levar a conseqüências mais perigosas como as quedas e fraturas, ${ }^{3}$ surgiu o interesse em desenvolver uma pesquisa que pudesse beneficiá-los, utilizando a equoterapia como recurso terapêutico, algo ainda não realizado nesse ciclo vital.

Após vários anos de estudos e pesquisas, a comprovação dos resultados levou o Conselho Federal de Medicina, em sessão plenária de 09/04/97, a reconhecer a equoterapia como um método terapêutico que utiliza o cavalo em uma abordagem interdisciplinar nas áreas de saúde, educação e equitação. O cavalo é o agente promotor de ganhos físicos e psicológicos e o terapeuta, o agente facilitador deste método. ${ }^{4}$ A equoterapia foi regulamentada também pelo Conselho Federal de 
Fisioterapia, em 2008, como um recurso terapêutico da Fisioterapia e Terapia Ocupacional. ${ }^{5}$

O uso da equoterapia surge como um recurso terapêutico que emprega o cavalo como motivador para o tratamento. Trata-se de um animal dócil, de porte e força, que se deixa manusear e montar. Dessa forma, o praticante e o cavalo criam um relacionamento afetivo importante, onde se estabelecem uma relação harmoniosa e atuação mútua.

Pela analogia de Ferreira, o cavalo possui ciclos de movimentação análogos aos ciclos do homem durante sua andadura natural, o passo. O paralelismo entre o andar humano e o cavalo é evidenciado pelo movimento tridimensional de ambos. Quando o cavalo se desloca, é exigido do praticante modular o tônus muscular para poder ajustar, manter, recuperar ou adaptar seu equilíbrio postural a cada movimento. ${ }^{5}$ Assim, ao analisar o movimento que o cavalo proporciona para o cavaleiro, almejou-se preencher as necessidades desses indivíduos, com a melhora do equilíbrio estático.

Segundo a American Hippotherapy Association, ${ }^{6}$ as indicações para a prática da equoterapia são: disfunções neuromusculoesqueléticas, alterações de tônus muscular, coordenação diminuída, comunicação inadequada, função sensório-motora alterada, assimetria postural, controle postural corporal, diminuição da atenção e distúrbios do comportamento. Sabendo disso e de muitos tratamentos bem-sucedidos na equoterapia em diversos pacientes, surgiu o interesse para desenvolver a pesquisa relacionando idosos com a equo- terapia e suas implicações sobre o equilíbrio dessa população.

Este trabalho teve por objetivo mensurar a melhora do equilíbrio estático em indivíduos da terceira idade com a prática da equoterapia. Procurou-se demonstrar as possibilidades de intervenção através dessa forma de tratamento, tendo o uso do cavalo como recurso terapêutico, proporcionando constante estimulação das estruturas e sistemas que comandam o equilíbrio e que, por conseguinte, levassem à melhora do controle postural.

\section{MATERIAS E MÉTODOS}

Estudo do tipo quase-experimental, préteste e pós-teste. A amostra foi composta por 10 indivíduos da terceira idade, correspondente a faixa etária de 60 a 74 anos, do sexo feminino, da cidade de Caxias do Sul, convidados aleatoriamente, no período de julho a novembro de 2007. Os critérios de exclusão da pesquisa foram: portadores de distúrbios neurológicos; indivíduos com diagnóstico de neoplasias; portadores de artroplastia e histórico de hipertensão arterial e/ ou diabetes mellitus não controlado e indivíduos que andaram a cavalo na meia idade, dos 45 aos 59 anos.

Para a análise do equilíbrio estático dos participantes da amostra foi utilizado o Estabilômetro AccuSway Plus, da marca AMTI (Advanced Mechanical Technology, INC USA), cujo software para a compreensão dos dados é o Balance Clinic, Balance Software by AMTI’s AccuSway Plus, Balance Plataform. 
Os indivíduos da terceira idade foram avaliados por meio do estabilômetro, no dia antes e um dia depois, nos mesmos horários, da aplicação do programa de atendimento equoterapêutico. As pessoas que avaliaram não foram as mesmas que aplicaram o programa.

Os testes foram realizados numa sala em ambiente silencioso, com temperatura de $24^{\circ} \mathrm{C}$, ajustada no ar-condicionado. Antes da realização do teste, os participantes ficaram sentados em repouso por cinco minutos. Durante o teste, o indivíduo foi solicitado a adotar a postura ortostática bipodal sobre a plataforma com os pés descalços. O espaço entre o segundo e o terceiro artelhos foi alinhado ao terceiro eixo lateralmente plotado na superfície da plataforma em relação ao eixo Y central (bilateralmente). O centro do arco longitudinal medial foi alinhado ao eixo X central da plataforma (bilateralmente). O indivíduo estava com os braços relaxados ao longo do corpo, olhando para um alvo a $90 \%$ da sua estatura, correspondente à altura dos olhos, a uma distância de $1,50 \mathrm{~m}$, onde permaneceu por cerca de 60 segundos. ${ }^{7,8}$

A equipe que realizou os atendimentos foi composta por um auxiliar-guia com a função de encilhar e guiar o cavalo; um fisioterapeuta e auxiliar lateral responsáveis pelo paciente e execução do programa; e três cavalos, exclusivamente utilizados para fins terapêuticos De uma sessão para a outra, os participantes alternaram os cavalos, e também usaram capacete para maior segurança.

As sessões de equoterapia foram realizadas no Centro de Equoterapia - EQUO-
CENTER, localizado no bairro Forqueta, em Caxias do Sul - RS. O programa de atendimento equoterapêutico constituiu-se de oito sessões de equoterapia, duas vezes por semana, com duração de 30 minutos cada.

Os resultados foram analisados na estabilometria, por meio dos valores obtidos de velocidade média de deslocamento do centro de pressão $(\mathrm{CP})$, deslocamento médio no eixo $\mathrm{X}$ do $\mathrm{CP}$ e deslocamento médio no eixo Y do CP. Também foi utilizado o teste estatístico t-Student pareado bicaudal. O nível de significância aceito foi de $\mathrm{p}<0,05$.

O estudo foi submetido ao Comitê de Ética em Pesquisa (CEP) da Universidade de Caxias do Sul (UCS) e aprovado, sob número 87/07. Um termo de consentimento livre e esclarecido foi assinado pelos participantes da pesquisa, conforme determina a Resolução no 196/96 do Conselho Nacional de Saúde (CNS). ${ }^{9}$

\section{RESULTADOS}

O primeiro parâmetro analisado (tabela 1) apresenta os valores do deslocamento no eixo $\mathrm{X}$ do $\mathrm{CP}$, correspondente ao sentido $\mathrm{M} / \mathrm{L}$, dos quais cinco dos indivíduos obtiveram melhora do equilíbrio estático. Salientam-se os indivíduos B e I, resultando numa diferença, entre o pré e o pós, de 56 e 55 pontos, respectivamente. No entanto, esses valores não foram suficientes para obter a diferença estatística significativa $(p=0,603)$. 
Tabela 1 - Valores do deslocamento no eixo X do CP, suas médias e desvio padrão. Caxias do Sul, RS, 2007.

\begin{tabular}{ccc}
\hline Indivíduos Participantes & $\begin{array}{c}\text { X Avg } \\
\text { Pré-Programa }\end{array}$ & $\begin{array}{c}\text { X Avg } \\
\text { Pós-Programa }\end{array}$ \\
\hline A & $-0,72$ & -1 \\
B & $-0,7$ & 0,14 \\
C & $-0,02$ & $-0,25$ \\
D & $-1,27$ & $-0,98$ \\
E & $-0,84$ & $-0,66$ \\
F & $-0,7$ & $-1,16$ \\
G & $-0,04$ & $-0,2$ \\
H & 0,59 & 0,64 \\
I & 0,56 & 0,01 \\
J & 0,31 & $-0,09$ \\
Médias & $-0,28$ & $-0,36$ \\
Desvio Padrão & 0,65 & 0,58 \\
\hline
\end{tabular}

As figuras 1, 2 e 3 demonstram as médias dos valores obtidos na estabilometria pré e pós-equoterapia.
$\mathrm{Na}$ figura 1, são apresentadas as médias dos deslocamentos no eixo $\mathrm{X}$ do $\mathrm{CP}$, as quais resultaram nos valores da pré-equoterapia $(-0,28)$ e pós-equoterapia $(-0,36)$.

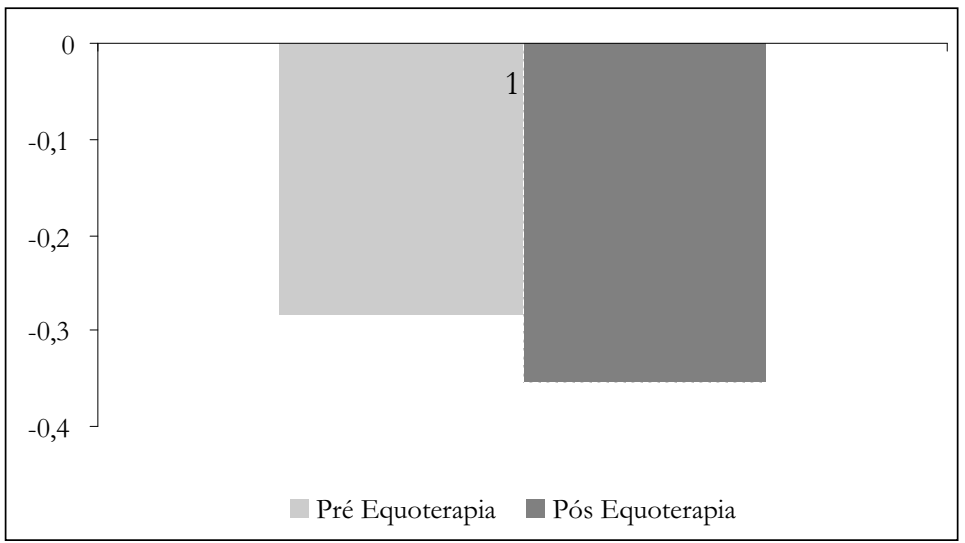

Figura 1 - Médias dos deslocamentos no eixo X do CP. 
$\mathrm{Na}$ tabela 2, os valores do deslocamento no eixo Y do CP demonstram que nove indivíduos apresentaram melhora do equilíbrio estático, especificamente a direção A/P. Assim sendo, por meio de suas médias, incluindo o décimo participante, obteve-se a estatística significante de $p=0,002$.

Tabela 2 - Valores do deslocamento no eixo Y do CP, suas médias e desvio padrão. Caxias do Sul, RS, 2007.

\begin{tabular}{ccc}
\hline Indivíduos Participantes & $\begin{array}{c}\text { Y Avg } \\
\text { Pré-Programa }\end{array}$ & $\begin{array}{c}\text { Y Avg } \\
\text { Pós-Programa }\end{array}$ \\
\hline A & $-0,08$ & 0,34 \\
B & $-0,75$ & $-0,12$ \\
C & $-1,25$ & 0,64 \\
D & $-0,74$ & $-0,37$ \\
E & $-0,96$ & $-0,38$ \\
F & $-1,14$ & $-0,34$ \\
G & $-1,62$ & $-0,91$ \\
H & $-1,28$ & $-0,97$ \\
I & $-1,34$ & $-1,21$ \\
J & $-1,41$ & $-0,37$ \\
Médias & $-1,06$ & $-0,37$ \\
Desvio Padrão & 0,44 & 0,57 \\
\hline
\end{tabular}

$\mathrm{Na}$ figura 2 são apresentadas as médias trando claramente a diferença entre o prédos deslocamentos no eixo Y do CP, mosequoterapia $(-1,06)$ e o pós-equoterapia $(-0,37)$.

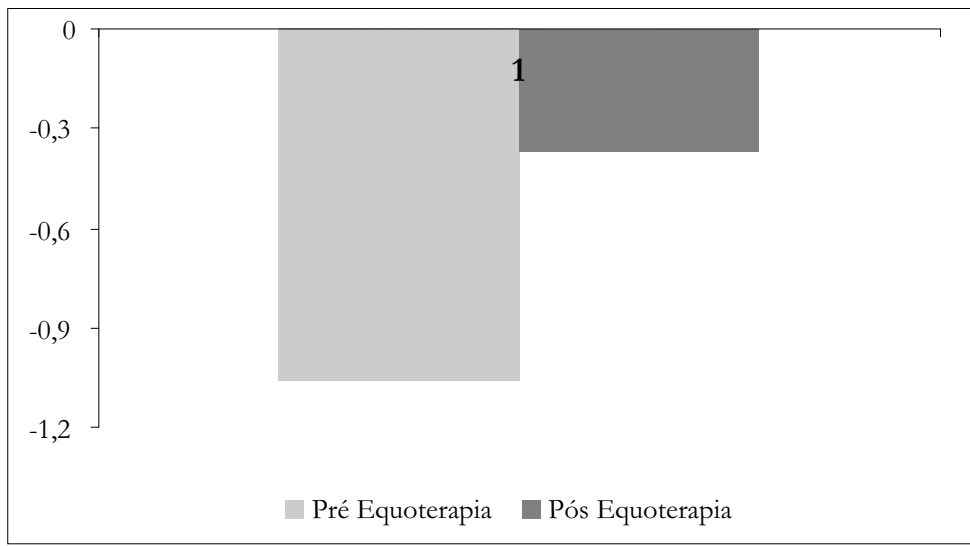

Figura 2 - Médias dos deslocamentos no eixo Y do CP. 
O último parâmetro analisado (tabela 3) demonstra as velocidades de deslocamento do $\mathrm{CP}$, em que sete indivíduos obtiveram melhora em seus valores. Mesmo com isso, o nível de significância estatística não foi alcançado $(p=0,414)$.

Tabela 3 - Valores da velocidade de deslocamento do CP, suas médias e desvio padrão. Caxias do Sul, RS, 2007.

\begin{tabular}{ccc}
\hline Indivíduos Participantes & $\begin{array}{c}\text { V Avg } \\
\text { Pré-Programa }\end{array}$ & $\begin{array}{c}\text { V Avg } \\
\text { Pós-Programa }\end{array}$ \\
\hline A & 0,52 & 0,5 \\
B & 0,59 & 0,55 \\
C & 0,65 & 0,6 \\
D & 0,66 & 0,61 \\
E & 0,72 & 0,69 \\
F & 0,62 & 0,73 \\
G & 0,52 & 0,53 \\
H & 0,73 & 0,56 \\
I & 0,58 & 0,64 \\
J & 0,58 & 0,56 \\
Médias & 0,62 & 0,60 \\
Desvio Padrão & 0,07 & 0,07 \\
\hline
\end{tabular}

A figura 3 demonstra as médias das velocidades de deslocamento do $\mathrm{CP}$, na qual a estabilometria resultou na pré-equoterapia $(0,62)$ e pós-equoterapia $(0,60)$.

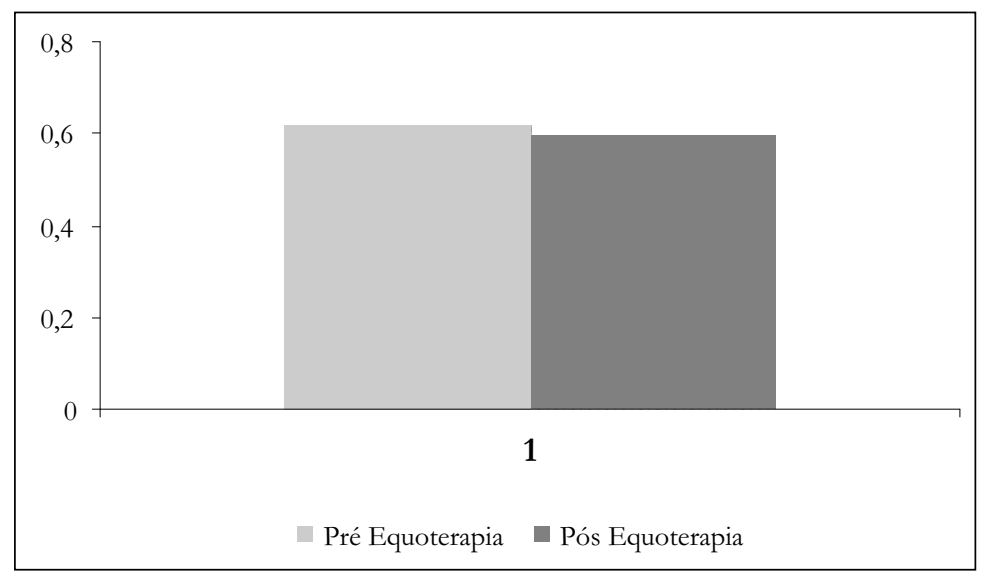

Figura 3 - Médias das velocidades de deslocamento do CP. 


\section{DISCUSSÃO}

Hoje, o desequilíbrio é um dos principais fatores que limitam a vida do idoso. As quedas são as conseqüências mais perigosas do desequilíbrio e da dificuldade de movimentação, sendo seguidas por fraturas, deixando os idosos acamados por dias ou meses. ${ }^{3}$ As manifestações dos distúrbios do equilíbrio corporal têm grande impacto para os idosos, podendo levá-los à redução de sua autonomia social, pela predisposição a quedas e fraturas, reduzindo suas atividades de vida diária, trazendo sofrimento, imobilidade, medo de cair novamente e altos custo com o tratamento de saúde. ${ }^{10}$

$\mathrm{Na}$ análise das médias dos deslocamentos no eixo X do CP (figura 1), é possível perceber que não houve diferença estatística significativa ( $\mathrm{p}=0,603)$. Isto ocorreu provavelmente porque os parâmetros mensurados na direção M/L são afetados pela base de apoio, sendo que aumentos na área de suporte refletem um decréscimo nos valores. ${ }^{11}$ A correlação negativa encontrada entre os parâmetros quantificados na direção $\mathrm{M} / \mathrm{L}$ e as bases de apoio pode ser explicada pelas propriedades biomecânicas do corpo. A mobilidade da articulação tíbio-társica (tornozelo) no plano frontal é reduzida com os pés separados. ${ }^{12}$

As atividades diárias envolvem adaptações das estratégias posturais sob diversas configurações de apoio, cuja estabilidade é constantemente modificada pela ativação muscular relativa às condições iniciais de apoio. ${ }^{13}$ Estratégias de controle postural com resposta motora aumentada sobre os tornozelos nos deslocamentos M/L são observadas com o aumento no ângulo de abertura e o afastamento dos calcanhares. ${ }^{14}$ Essa abertura da base de apoio reforça a coordenação dos movimentos entre os tornozelos e quadris, aumentando a sensibilidade para os movimentos laterais. ${ }^{15}$

No presente estudo, o protocolo de tempo utilizado na estabilometria foi de 60 segundos. Esse período é recomendado para assegurar a estacionariedade do sinal estabilométrico. ${ }^{7,8}$ No entanto, por meio do resultado não-significativo do deslocamento lateral, recomenda-se adaptar tempos maiores de avaliação ao determinado grupo da pesquisa. Ao relatar isso, cita-se o estudo de Imbiriba e colaboradores,${ }^{16}$ no qual se observou uma relação entre o deslocamento lateral e a sensação de desconforto, quando se compararam resultados de indivíduos que permaneceram continuamente de pé sobre a plataforma com os de outros que repousaram durante o teste, a partir do $15^{\circ}$ minuto. Como os próprios autores relatam, a permanência da postura prolongada de pé manifestou um alto nível de desconforto nos indivíduos, atribuído a um processo de fadiga. Então, reforça-se a idéia de adequar o tempo em conformidade com o público em estudo.

No segundo parâmetro avaliado, ao verificar as médias dos deslocamentos no eixo $\mathrm{Y}$ do CP (figura 2), depara-se com diferença estatística significativa $(p=0,002)$. Ao utilizar o protocolo de pés separados, o deslocamento do CP tende a ser maior na direção A/P. ${ }^{12,15,17}$ Em conformidade a isso, pode-se comprovar a eficácia do uso da equoterapia como 
recurso terapêutico para a melhora do equilíbrio estático na direção ântero-posterior em indivíduos da terceira idade, na qual os valores decresceram na estabilometria pré para a pós-equoterapia.

A característica mais importante para a equoterapia é a andadura ao passo. Ele produz no cavalo e transmite ao cavaleiro uma série de movimentos seqüenciados e simultâneos, que tem como resultante o movimento tridimensional ou multidirecional. O mesmo se traduz no eixo vertical, em movimento para cima e para baixo; no plano frontal, em movimento para a direita e para a esquerda; e no plano sagital, em movimento para frente $\mathrm{e}$ para trás. A esses três movimentos, associa-se um quarto provocado pelo cavalo, que é uma torção da bacia do cavaleiro da ordem de até oito graus para cada lado, provocado pelas inflexões laterais do dorso do animal. ${ }^{18}$

O passo completo do cavalo corresponde ao deslocamento de seus quatro membros no solo. Em um passo, o cavaleiro é estimulado duas vezes infra-superiormente, tem-se dois deslocamentos laterais, um para a esquerda e outro para a direita, deslocado duas vezes ântero-posteriormente, e executa o movimento de rotação da pelve bilateralmente. ${ }^{18}$

O cavalo realiza em média 50 passos por minuto. Sendo assim, proporciona 150 ondulações tridimensionais por minuto, o que origina 450 correções posturais no praticante, e ativando 900 grupos musculares. ${ }^{19}$ Ao final de uma sessão de 30 minutos, equivale a 216.000 contrações musculares, desde que não seja feito nenhum exercício adicional.
Com o passar dos anos, o organismo humano passa por um processo natural de envelhecimento. Ruwer et al. ${ }^{10}$ relatam que esse envelhecimento compromete a habilidade do sistema nervoso central em realizar o processamento dos sistemas vestibulares, visuais e proprioceptivos responsáveis pela manutenção do equilíbrio corporal. Quando esses sistemas não são integrados corretamente, origina-se uma perturbação do estado de equilíbrio, que pode ser manifestada por desequilíbrio corporal, podendo culminar com o evento da queda. ${ }^{20}$ Entretanto, todos os sistemas de nosso organismo possuem reservas fisiológicas, que no sistema nervoso são caracterizadas pela capacidade de reorganização, conhecida como neuroplasticidade. ${ }^{21}$ Com o envelhecimento, as reservas estão diminuídas, porém não depletadas; portanto, a criação de um ambiente ideal de aprendizado motor poderia determinar uma melhora importante da função. ${ }^{22}$ Sendo assim, o resultado adquirido na pesquisa vem a confirmar essa teoria e justificar a importância do uso da equoterapia como recurso terapêutico para a melhora do equilíbrio estático em indivíduos da terceira idade.

A cada passo do cavalo, o centro de gravidade do praticante é defletido da linha média, estimulando as reações de equilíbrio. O sistema vestibular é assim repentinamente solicitado, estimulando continuamente suas conexões entre os canais semicirculares, onde as células ciliares e os otólitos captam as oscilações da endolinfa provocadas pelos movimentos da cabeça através do cerebelo, tálamo, córtex cerebral, medula espinhal e nervos periféricos, em ambos os sentidos - ascendente e descendente. ${ }^{23}$ 
Conforme as médias das velocidades de deslocamento do CP (figura 3), o nível de significância estatística não foi alcançado $(\mathrm{p}=0,414)$. Isso pode ser explicado pela realização do protocolo de estabilometria com os olhos abertos. Justifica-se isso pelo estudo de Gandra et al., ${ }^{24}$ os quais verificaram, em um grupo de indivíduos jovens, o aumento da área elíptica e da velocidade média de deslocamento ao longo do tempo, com a privação da informação visual, iniciando no $10^{\circ}$ minuto de teste. Com a diminuição do tamanho da base de apoio, ocorre o aumento da velocidade de deslocamento. ${ }^{15}$

A contribuição da visão no controle postural durante a posição ortostática pode ser facilmente demonstrada pelo simples fechar dos olhos, gerando, na maioria dos indivíduos, aumento nos valores dos parâmetros do $\mathrm{CP}$ de duas a três vezes em relação a OA. ${ }^{25,26}$ Os déficits visuais, na maioria das vezes, estão associados aos riscos de queda, principalmente em idosos, pois a contribuição da visão no controle postural aumenta com a idade, sendo prejudicada na presença de perturbações visuais ou doenças da retina. ${ }^{27,28}$

As oscilações do CP estudadas com base de apoio de dimensões diversas demonstram que a rapidez com que o corpo se move aumenta ao diminuir a base de apoio, ou na condição olho fechado (OF). A interação entre esses dois fatores pode ser explicada pela presença da visão no controle postural, pois a visão reduz eficientemente os movimentos corporais quando os pés estão posicionados juntos. ${ }^{15}$ Investigação com dois grupos etários distintos, abaixo de 45 anos e acima de 45 anos, foi conduzida por Accornero et al., ${ }^{29}$ que estudaram as estratégias do controle das oscilações corporais com protocolo de pés ladoa-lado, com OA e OF. Esses autores encontraram diferenças significativas $(\mathrm{p}<0,001)$ entre OA e OF, com maior oscilação corporal ocorrendo durante $\mathrm{OF}$ e prevalecendo no grupo mais velho.

$\mathrm{Na}$ atual pesquisa, ao saber da existência de usuários de lentes corretivas entre os participantes e da influência que a visão proporciona no controle postural, sugere-se a necessidade da avaliação na estabilometria com OF, podendo com isso excluir a variável do déficit visual, que porventura possam ter os participantes. Em alguns casos, o indivíduo pode apresentar desequilibrios, não necessariamente atribuído ao sistema de equilíbrio como um todo, e sim ao sistema visual. ${ }^{30,31}$

A atividade física na terceira idade proporciona muitos benefícios à saúde. Um dos benefícios atribuídos é a melhora do equilíbrio postural. Sendo assim, ao verificar os critérios de exclusão desta pesquisa, deparouse com a não-inclusão desse fator. Entretanto, por meio dos resultados obtidos no estudo, surpreendeu-se que mesmo indivíduos praticantes de atividades físicas possam melhorar ainda mais o equilíbrio postural.

$\mathrm{Na}$ literatura, não há consenso sobre o número adequado de sessões de tratamento necessárias para obter melhora do equilíbrio estático. Assim sendo, no programa de atendimento equoterapêutico desenvolvido para este estudo, utilizaram-se oito sessões de equoterapia. Para a comprovação da melhora do 
equilíbrio estático ântero-posterior, o número proposto foi suficiente. Por outro lado, conforme os resultados do equilíbrio médiolateral e velocidade de deslocamento sugeremse mais sessões.

Outro aspecto a ser apresentado refere-se aos resultados subjetivos do estudo, que não foi objetivo inicial, mas que merece destaque. Por meio do diário de campo, foi registrada a percepção das voluntárias sobre o movimento que o cavalo transmite ao praticante, sua dificuldade em manter o equilíbrio ao realizar o exercício parar e andar, as dores musculares nas pernas após as primeiras sessões, a mansidão dos animais, a confiança adquirida com o tempo, a consciência corporal ao verificar o tamanho dos olhos dos cavalos e o bem-estar que o ambiente proporciona. A equoterapia oferece a interação com o animal, a equipe, o espaço utilizado, os elementos da natureza, os cuidados preliminares, a montaria e o manuseio final, desenvolvendo novas formas de socialização, maior confiança e aumento da auto-estima. ${ }^{23}$

\section{CONCLUSÃO}

Os resultados encontrados nesta pesquisa demonstraram, através da estabilometria, que os indivíduos da terceira idade saudáveis possuem alterações no equilíbrio e, desta forma, correm o risco de sofrer quedas. Entretanto, o uso da equoterapia, aplicada conforme descrito nos procedimentos, foi capaz de melhorar o equilibrio estático nesta amostra e, conseqüentemente, diminuir a possibilidade de queda.
Por meio do resultado obtido no estudo, o deslocamento no eixo $\mathrm{Y}$ do $\mathrm{CP}$ confirma a teoria de Umphred, ${ }^{22}$ que diz que a criação de um ambiente ideal de aprendizado motor pode determinar uma melhoria importante da função. A repetição de uma tarefa é seguida de aprendizado, levando a uma melhoria de desempenho da atividade motora. ${ }^{32}$ Assim, a redução da oscilação corporal por aprendizado é conseqüência da diminuição das ações necessárias para a ativação muscular que mantém o corpo estável junto à base de suporte.

Indivíduos da terceira idade que relatam ou não a presença de desequilíbrios ou dificuldade de locomoção e/ ou o evento de quedas devem praticar atividades para estimular o sistema vestibular, atividades lúdicas e muito prazerosas para quem as pratica, e que tenham o caráter de ser preventivas e curativas em relação às alterações do equilíbrio e ao risco de quedas.

Considerando que a queda é um evento que modifica substancialmente a qualidade de vida do idoso e que a expectativa de vida da população em geral tem aumentado significativamente, fazendo com que a porcentagem de população idosa aumente a cada ano, condutas terapêuticas gerais voltadas ao idoso e especialmente aquelas que visem à prevenção da queda pela melhora da estabilidade postural culminarão na melhoria da qualidade de vida desta parcela da população. Sendo assim, a equoterapia parece ser um recurso terapêutico eficaz na melhora do equilíbrio de indivíduos da terceira idade. 
Ao retomar o protocolo utilizado na estabilometria e os resultados estatísticos não-significativos do deslocamento no eixo $\mathrm{X}$ do $\mathrm{CP}$ e da velocidade de deslocamento do CP, sugere-se adequar ao grupo em estudo e aos parâmetros a serem analisados, o posicionamento dos pés sobre a plataforma de força, olhos abertos e/ou fechados e tempos maiores que 60 segundos.

\section{REFERÊNCIAS}

1. Instituto Brasileiro de Geografia e Estatística - IBGE, 2007. População Segundo os Grupos de Idade. [acesso 2007 out 15]. Disponível em: URL: http:// www.ibge.gov.br/home/estatística/ população/censo2000/ default_tabulacao.shtm.

2. Geneher CA. A utilização de técnicas de Equoterapia e o nível de independência dos indivíduos de terceira idade. In: Coletânea de Trabalhos do $2^{\circ}$ Congresso Brasileiro de Equoterapia, 2002; São Paulo. São Paulo, 2002.

3. Bittar RSM, Pedallini MEB, Bottino MA, Formigoni LG. Síndrome do desequilíbrio no idoso. Pró-Fono. 2002; 14 (1):119-28

4. Cirillo LC. Fundamentos doutrinários da Equoterapia no Brasil. In: Coletânea de Trabalhos do II Congresso Brasileiro de Equoterapia. São Paulo, 2002.

5. COFFITO. Jornal Eletrônico [acesso 2008 out 12] Disponível em: http:// www.coffito.org.br/arq_sys/download/ JORNAL_COFFITO_eletr\%oC3\%B4nico.pdf

6. AHA (American Hippoterapy Association). Precauções e contra-indicações absolutas para a Equoterapia INCOMPLETA http:/ / www.americanhippotherapyassociation.org/

7. Carpenter M, Frank JS, Winter DA, Peysar GH. Sampling duration effects on centre of

\section{NOTAS}

Fisioterapeuta, graduado pela Universidade de Caxias do Sul.

b Mestre em Engenharia Biomédica, Universidade do Vale do Paraíba. Professor do curso de Fisioterapia da Universidade de Caxias do Sul.

c Mestre em Ciências do Movimento Humano, Universidade Federal do Rio Grande do Sul. Professora do Curso de Fisioterapia da Universidade de Caxias do Sul.

pressure summary measures. Gait Posture 2001; 13 (1): 35-40

8. Mello RGT, Gandra VD, Oliveira LF, Nadal J. Influência do tempo de aquisição nos parâmetros do sinal estabilométrico. Anais do $18^{\circ}$ Congresso Brasileiro de Engenharia Biomédica. São José dos Campos, 2002. p. 406-10

9. Conselho Nacional de Saúde. Resolução n.196, de 10 de outubro de 1996. Brasília (DF): Conselho Nacional de Saúde, 1996.

10. Ruwer SL, Rossi AG, Simon LF. Equilíbrio no Idoso. Rev Bras Otorrinolaringol 2005; 71 (3): 293-303

11. Chiari L, Rocchi L, Cappello A. Stabilometric parameters are affected by anthropometry and foot placement. Clin Biomech 2002; 17 (9-10): 666-77

12. Kirby RL, Price NA, Macleod DA. The Influence of Foot Position on Standing Balance. J Biomech 1987; 20 (4): 423-427

13. Henry SM, Fung J. Horak FB. Effect of stance width on multidirectional postural responses. J Neurophysiol 2001; 85: 559-70

14. Prince F, Winter DA, Archer SF. Assessment of postural control during quiet stance with different foot configuration. Gait Posture. 1995; 3 (2): 110-1. 
15. Day BL, Steiger MJ, Thompson PD, Marsden CD. Effect of vision and stance width on human body motion when standing: implication for afferent control of lateral sway. J Physiol 1993; 469: 479-99

16. Imbiribiba LA, Cruz CR, Mainentini MR, Magalhães J, Garcia MAC, Oliveira LF et al. Modification in the postural control during long-term standing. Proceedings of the $4^{\text {th }}$ World Congress of Biomechanics. Calgary CA, 2002.

17. McIlroy WE, Maki BE. Preferred placement of the feet during quiet stance: development of a standardized foot placement for balance. Clin Biomech 1997, 12 (1): 66-70

18. Medeiros M, Dias E. Equoterapia: bases e fundamentos. Rio de Janeiro: Revinter; 2002.

19. Walter GB, Vendramini OM. Equoterapia: terapia com o uso do cavalo. Viçosa, MG: CPT/CEE-UFV; 2000.

20. Barbosa SM, Arakaki J, Silva MF. Estudo do equilíbrio em idosos através da fotogrametria computadorizada. Fisioterapia Brasil 2001; 2 (3): 187-96

21. Piovesana AMSG. Plasticidade cerebral aspectos clínicos. Arq Neuropsquiatr 2001; 59 (suppl 1): 17-9

22. Umphred D, Lewis RW O envelhecimento e o sistema nervoso central. In: Kauffman TL (editor). Manual de reabilitação geriátrica. Rio de Janeiro: Guanabara Koogan; 2001.

23. Ganança MM, Munhoz MSL. Equilibriometria clínica. São Paulo: Atheneu; 1999.

24. Gandra VD, Oliveira LF, Nadal JB. Efeito da visão no controle postural ortostático em testes estabilométricos de longa duração.
Anais do X Congresso Brasileiro de

Biomecânica, Ouro Preto, 2003. v. 2, p. 430-3.

25. Chiari L, Cappello A, Lenzi D, Della Croce U. An Improved Technique for the Extraction of Stochastic Parameters from Stabilograms. Gait Posture 2000, 12 (3): 225-34

26. Kelly JW, Loomis JM, Beall AC. The importance of perceived relative motion in the control of posture. Exp Brain Res 2005, 161 (3): 285-92

27. Straube A, Krafczyk S, Paulus W, Brandt T. Dependence of visual stabilization of postural sway on the cortical magnification factor of restricted visual fields. Exp Brain Res 1994, 99 (4): 501-6.

28. Turano KA, Dagnelie G, Herdman SJ. Visual stabilization of posture in persons with central visual field loss. Invest Ophthalmol Vis Sci 1996; 37 (8): 1483-91

29. Accornero N, Capozza M, Rinalduzzi S, Manfredi GW. Clinical multisegmental posturography: age-related changes in stance control. Electroencephalogr Clin Neurophysiol 1997; 105 (3): 213-19.

30. Chew PTK. Measurement of the visual contribution to postural steadiness from the cop movement: methodology and reliability. Gait Posture 2004; 22 (2): 96-106.

31. Chiari L, Bertani A, Cappello A Classification of visual strategies in human postural control by stochastic parameters. Hum Mov Sci 2000; 19 (6): 817-42

32. Tarantola J, Nardone A, Tacchini E, Schieppati M. Human stance stability improves with the repetition of task: effect of foot position and visual condition. Neurosci Lett 1997; 228 (2): 75-8. 
1 